\title{
Le financement informel dans l'agriculture algérienne : les principales pratiques et leurs déterminants*
}

\author{
Ali Daoudi ${ }^{1}$ \\ Betty Wampfler ${ }^{2}$ \\ ${ }^{1}$ Institut national agronomique \\ École nationale supérieure agronomique \\ Département d'économie rurale \\ Hacène Badi - El Harrach \\ 16200 Alger \\ Algérie \\ <daoudinf@yahoo.fr> \\ 2 IRC SupAgro Montpellier \\ 1101, Avenue Agropolis \\ BP 5098 \\ 34033 Montpellier \\ France \\ <betty.wampfler@supagro.inra.fr>
}

\begin{abstract}
Résumé
Le financement informel, pratique courante dans le secteur agricole en Algérie, reste un phénomène peu étudié. Cet article présente des résultats d'une étude ayant pour objectifs de caractériser cette pratique et d'explorer ses déterminants structurels dans quatre régions agricoles du pays. Quatre principaux types de pratiques y sont identifiés mais dans des proportions qui varient avec les caractéristiques structurelles de ces régions : la vente sur pied avec préfinancement, l'association de production, le crédit fournisseur et le prêt entre particuliers. D'ordre naturel et socio-économique, ces caractéristiques confèrent leurs spécificités aux territoires agricoles, déterminent les opportunités de financement informel offertes aux agriculteurs et influencent leurs choix.
\end{abstract}

Mots clés : Algérie ; crédit agricole ; financement ; système d'exploitation agricole.

Thèmes : économie et développement rural ; systèmes agraires.

\section{Abstract \\ Informal financing of Algerian agriculture: Main practices and determinants}

Informal funding in the agricultural sector of Algeria remains a subject receiving limited attention. This article presents the results of an empirical study aimed at characterizing the informal financing practices and exploring their structural determinants in four agricultural regions of the country. The study identified four main types of practices: standing crop sale with pre-financing, combined production, supplier credit and lending between individuals. These practices are present in all four regions, but in different proportions, related to the structural characteristics of the regions. Natural and socio-economic characteristics result in farming specificities, condition the informal funding opportunities offered farmers and influence their choices.

Key words: agricultural credit; Algeria; farming systems; funding.

Subjects: economy and rural development; farming systems.
E n Algérie, comme dans de nombreux pays en développement, les réformes des systèmes financiers n'ont pas abouti à l'émergence de marchés financiers ruraux dynamiques pouvant répondre efficacement aux besoins de toutes les catégories d'exploitations agricoles. En Afrique et en Asie du Sud, le taux de bancarisation de l'agriculture ne dépasse par 6\% (Morvant-Roux, 2008). Dans beaucoup de ces pays, 50 à $80 \%$ des habitants ont un problème d'accès à des services financiers adéquats (Banque mondiale, 2007). Dans les pays du Maghreb, la situation n'est pas meilleure. En Tunisie, la proportion des exploitations agricoles accédant au financement bancaire atteint à peine $10 \%$ (Audinet Tunisie, 2007). Au Maroc, le secteur agricole souffre d'un sousfinancement important et les banques ne contribuent que très peu dans le financement des besoins des exploitations (Akesbi et al., 2008). Le même caractère

\footnotetext{
* Pour citer cet article : Daoudi A, Wampfler B. Le financement informel dans l'agriculture algérienne : les principales pratiques et leurs déterminants. Cah Agric $2010 ; 19$ : 243-8. doi : 10.1684/agr.2010.0414
} 
marginal de la contribution des banques au financement des exploitations agricoles est relevé en Algérie (Conseil national économique et social, 2003 ; Mesli, 2007).

Le manque d'information sur les détails opérationnels des exploitations, leur incapacité à fournir des garanties matérielles et l'importance des coûts de transaction seraient les principales raisons de cette exclusion (Ruan et Zhang, 2008 ; Andersson, 2008). Dans les pays du Maghreb, la frilosité des banques à l'égard du secteur agricole est accentuée par l'importance des risques climatiques qui rendent la production très aléatoire (Ben El Ahmar, 2003 ; Mesli, 2004).

L'importance du crédit agricole dans la modernisation de l'agriculture de pays comme l'Algérie remet à l'ordre du jour les réflexions sur les conditions qui rendent ce crédit accessible aux agriculteurs. L'étude du fonctionnement des pratiques de financement informel, qui seraient très fréquentes dans l'agriculture algérienne (Daoudi et Bédrani, 2002, 2008 ; CNES, 2003), peut dégager des pistes de réflexion susceptibles d'alimenter le débat sur la réforme du système de financement agricole. Cet article présente les résultats d'une recherche empirique sur le financement informel dans le secteur agricole algérien. Après définition du cadre conceptuel et méthodologique, il décrit les principales formes de financement informel pratiquées et propose une interprétation de leurs déterminants structurels.

\section{Cadre conceptuel et méthodologie}

Le financement informel est un trait caractéristique des économies agricoles et rurales des pays en développement (Adams et Fitchett, 1994 ; Tsai Kelee, 2004 ; Maksimovic et al., 2008 ; Andersson, 2008). En Algérie, même si son existence est généralement admise, il reste une facette inexplorée de la réalité de l'agriculture. En l'absence de connaissances empiriques validées, l'identification des principales formes de financement informel a été le premier enjeu de ce travail. Considérant le financement informel comme étant " toute transaction qui permet aux agriculteurs de mobiliser des moyens productifs auprès d'un tiers parti non formel ", l'étude s'est attachée à analyser les deux termes de cette transaction: les besoins des exploitations agricoles et leurs sources de financement dans des zones agricoles sup- posées représenter différentes situations d'offre. Car nous considérons que les pratiques de financement informel des agriculteurs seraient déterminées non seulement par les besoins en financement, eux-mêmes déterminés par la nature des systèmes de production, mais aussi par l'offre informelle qui est déterminée à son tour par des facteurs économiques (Venet, 1994) et socioculturels (Henni, 1993 ; Servet, 1995). Dans une synthèse de ses travaux sur l'économie géographique, Krugman (2004), montre clairement la relation directe qui existe entre la taille du marché et la localisation de l'offre. En nous appuyant sur cette thèse, nous considérons que la configuration de l'offre de financement informel varie avec la configuration de l'économie agricole de chaque région du pays. Ainsi, la concentration de l'offre de financement informel serait liée à la concentration d'une spéculation agricole donnée, ellemême déterminée par les paramètres agroécologiques.

Quatre wilayas sont retenues pour représenter les principales zones agroécologiques du pays : Blida pour les plaines littorales, Constantine pour les hautes plaines intérieures, Djelfa pour les zones steppiques et Tlemcen pour les zones de montagne. Dans chacune de ces wilayas, un échantillon de 100 agriculteurs a été enquêté en 2002.

\section{Pratiques}

\section{de financement informel : caractéristiques et importance relative}

Les agriculteurs mobilisent quatre sources de financement pour satisfaire les besoins financiers de leur système de production. L'autofinancement en est de loin la principale. Les subventions publiques sont aléatoires, ne touchant que quelques spéculations alors que le crédit bancaire est insignifiant $(0,75 \%$ des enquêtés y ont recouru durant la campagne considérée). Le financement informel est, en revanche, fortement développé et organisé autour de quatre types de pratiques: la vente sur pied avec préfinancement, l'association de production, le crédit fournisseur et le prêt entre particuliers. Chacune de ces formes répond à un besoin spécifique et est mobilisée par un type particulier d'agriculteurs. L'offre de financement émane d'acteurs de l'environnement de l'agriculteur poursuivant des fins économiques ou sociales. Le tableau 1 montre la diversité des caractéristiques des pratiques et les principes qui les sous-tendent. La quantification précise de l'importance relative de ces formes de financement a été impossible du fait de la difficulté de collecte de données sur les montants monétaires mobilisés. Aussi, leur importance a été appréhendée à travers la fréquence de leur mobilisation par les agriculteurs et l'étude s'est concentrée sur l'analyse des processus qui les permettent.

\section{La vente sur pied avec préfinancement}

Le contrat classique de vente sur pied d'une récolte donnée est ici élargi par des clauses spécifiant la mobilisation, par l'acheteur, de ressources financières pour couvrir tout ou partie des charges de campagne. Cette mobilisation peut prendre la forme d'une avance sur le montant de l'achat de la récolte ou celle d'une contribution de l'acheteur dans la prise en charge directe de dépenses de production. Les arrangements sont multiples et concernent surtout le partage des responsabilités techniques et financières de la conduite du processus de production. La négociation et la conclusion de ce type de transaction se font au début du cycle biologique de la production concernée.

Cette pratique, exclusivement dédiée à l'arboriculture fruitière chez les attributaires des terres des ex-domaines publics, n'est rencontrée qu'à Blida, l'une des principales wilayas arboricoles du pays, où $56 \%$ des enquêtés couvrent les charges d'acquisition des intrants agricoles grâce aux avances des commerçants collecteurs.

\section{L'association de production}

Ce type de transaction permet à des individus disposant de facteurs de production différents mais complémentaires de les combiner et de les faire fructifier dans le cadre de processus de coproduction. Le propriétaire de la terre donne celle-ci à un tiers, porteur d'un capital, pour la travailler en association ou la travaille lui-même en association avec la contribution d'un partenaire qui apporte le capital. Le partage de la récolte entre associés se fait proportionnellement à l'apport de chacun en facteurs de production.

L'association de production concerne généralement des cultures annuelles, 
Tableau 1. Principales caractéristiques des formes de financement informel pratiquées par les agriculteurs.

Table 1. Principal characteristics of farmers' informal funding practices.

\begin{tabular}{|c|c|c|c|c|c|}
\hline \multirow{2}{*}{$\begin{array}{l}\text { Formes de financement } \\
\text { informel }\end{array}$} & \multicolumn{5}{|c|}{ Principales caractéristiques } \\
\hline & $\begin{array}{l}\text { Objet principal } \\
\text { de la transaction }\end{array}$ & $\begin{array}{c}\text { Type de service } \\
\text { financier }\end{array}$ & $\begin{array}{l}\text { Bénéficiaires } \\
\text { potentiels }\end{array}$ & $\begin{array}{c}\text { Offreurs } \\
\text { potentiels }\end{array}$ & Intérêt de l'offreur \\
\hline $\begin{array}{l}\text { Vente sur pied } \\
\text { avec préfinancement }\end{array}$ & $\begin{array}{l}\text { Récolte sur } \\
\text { pied de fruits } \\
\text { et légumes }\end{array}$ & $\begin{array}{l}\text { Préfinancement } \\
\text { ou avance } \\
\text { sur le montant } \\
\text { de la transaction } \\
\text { de vente }\end{array}$ & $\begin{array}{l}\text { Les arboriculteurs } \\
\text { des zones } \\
\text { potentielles, } \\
\text { notamment } \\
\text { les attributaires } \\
\text { des terres des ex- } \\
\text { domaines publics }\end{array}$ & $\begin{array}{l}\text { Les commerçants } \\
\text { collecteurs livreurs } \\
\text { des fruits } \\
\text { et légumes }\end{array}$ & $\begin{array}{l}\text { Négociation, de gré } \\
\text { à gré, d'un prix } \\
\text { d'achat préférentiel }\end{array}$ \\
\hline $\begin{array}{l}\text { L'association } \\
\text { de production }\end{array}$ & Coproduction & $\begin{array}{l}\text { Apport } \\
\text { d'un facteur } \\
\text { de production } \\
\text { (terre ou capital) }\end{array}$ & $\begin{array}{l}\text { Les propriétaires } \\
\text { terriens sans capital } \\
\text { Les exploitants } \\
\text { agricoles, sans terres }\end{array}$ & $\begin{array}{l}\text { Les exploitants } \\
\text { agricoles, sans } \\
\text { terres } \\
\text { Les propriétaires } \\
\text { terriens sans } \\
\text { capital }\end{array}$ & $\begin{array}{l}\text { Part } \\
\text { de la production } \\
\text { réalisée, } \\
\text { valorisation } \\
\text { d'un facteur } \\
\text { de production } \\
\text { possédé }\end{array}$ \\
\hline Le crédit fournisseur & $\begin{array}{l}\text { Intrants } \\
\text { ou services } \\
\text { agricoles } \\
\text { Dans une } \\
\text { moindre } \\
\text { mesure, } \\
\text { acquisition } \\
\text { de matériel } \\
\text { agricole } \\
\text { ou réalisation } \\
\text { de forages }\end{array}$ & $\begin{array}{l}\text { Crédit de } \\
\text { paiement }\end{array}$ & $\begin{array}{l}\text { Pour le crédit relatif } \\
\text { aux services : } \\
\text { Les céréaliculteurs } \\
\text { potentiels } \\
\text { Les éleveurs } \\
\text { potentiels } \\
\text { Pour le crédit } \\
\text { relatif à l'achat } \\
\text { d'intrants: } \\
\text { Les maraîchers } \\
\text { potentiels } \\
\text { Les éleveurs } \\
\text { potentiels } \\
\text { Les aviculteurs } \\
\text { potentiels }\end{array}$ & $\begin{array}{l}\text { Les professionnels } \\
\text { de la location } \\
\text { de matériel } \\
\text { agricole } \\
\text { Les fournisseurs } \\
\text { des services } \\
\text { et produits } \\
\text { vétérinaires } \\
\text { Les fournisseurs } \\
\text { d'intrants agricoles } \\
\text { Les fournisseurs } \\
\text { d'aliments } \\
\text { du bétail } \\
\text { et des volailles } \\
\text { Les revendeurs } \\
\text { de matériel } \\
\text { agricole } \\
\text { Les entreprises } \\
\text { de réalisation } \\
\text { de forages }\end{array}$ & $\begin{array}{l}\text { Fidélisation } \\
\text { des clients } \\
\text { potentiels } \\
\text { et ou amélioration } \\
\text { du chiffre d'affaire }\end{array}$ \\
\hline $\begin{array}{l}\text { Le prêt } \\
\text { entre particuliers }\end{array}$ & Argent liquide & $\begin{array}{l}\text { Prêt } \\
\text { non rémunéré }\end{array}$ & $\begin{array}{l}\text { Tous } \\
\text { les agriculteurs }\end{array}$ & $\begin{array}{l}\text { Parents, amis } \\
\text { et partenaires } \\
\text { économiques }\end{array}$ & $\begin{array}{l}\text { Méta-financier } \\
\text { (solidarité, } \\
\text { réciprocité...) }\end{array}$ \\
\hline
\end{tabular}

maraîchage et céréaliculture, et s'observe également en aviculture. Près de $38 \%$ des enquêtés des quatre wilayas y ont recouru au moins une fois durant la période 19972002. Pour la campagne 2001-2002, $18 \%$, $18 \%, 14 \%$ et $1 \%$ des enquêtés respectivement à Blida, Tlemcen, Constantine et Djelfa, ont pratiqué l'association de production pour au moins une spéculation agricole, $43 \%$ des contrats recensés concernant les cultures maraîchères et plus de $39 \%$ les céréales.

\section{Le crédit fournisseur d'intrants et de services agricoles}

Dans ces transactions, des fournisseurs d'intrants ou de services agricoles accordent à des clients des facilités de paiement de leurs acquisitions en services et/ou en intrants. Le crédit fournisseur peut être mobilisé, mais dans une moindre mesure, pour le financement d'investissements productifs.
Plus de $71 \%$ des enquêtés ont bénéficié, au moins une fois, d'un crédit fournisseur durant la période 1997-2002. Ce taux varie d'une wilaya à une autre : il est de $82 \%$ à Djelfa, de $80 \%$ à Constantine, $72 \%$ à Tlemcen et de près de $61 \%$ à Blida.

\section{Le prêt entre particuliers}

Les prêts entre particuliers se font presque exclusivement en argent et ce, sans inté- 
rêt. Parmi les enquêtés, 72 \% ont recouru au moins une fois à un emprunt auprès de particuliers pour financer une activité agricole productive durant la période 1997-2002. Dans environ $84 \%$ des cas, ils en ont disposé pour une période allant de quelques semaines à quelques mois. La mobilisation de ces prêts n'est pas limitée à un type particulier de besoins et peut financer des charges courantes ou des investissements productifs. Ainsi, 11,6\% des investissements agricoles productifs réalisés durant la période 1997-2002 ont été financés grâce à des emprunts contractés chez des particuliers. Quant aux montants concernés, ils sont inférieurs ou égaux, dans plus de $72 \%$ des cas, à $100000 \mathrm{DA}^{1}$.

\section{Déterminants}

\section{des pratiques}

\section{de financement informel dans l'agriculture}

Les formes de financement informel identifiées sont, presque toutes, présentes dans chacune des régions étudiées, mais dans des proportions différentes, une à deux d'entre elles étant plus fréquentes que les autres.

Â Blida, le secteur agricole se caractérise par l'importance de la superficie irriguée qui atteint $38 \%$ de la SAU de la wilaya et qui est surtout consacrée à l'arboriculture fruitière et dans une moindre mesure au maraîchage. Cette wilaya totalise à elle seule $27 \%$ du verger agrumicole national avec 12754 hectares (Recensement général de l'agriculture (RGA), 2003). Elle compte aussi les plus grands marchés de gros de fruits et légumes du pays, les commerçants étant les véritables banquiers de la filière fruits et légumes de toute la wilaya.

Les pratiques de financement informel les plus fréquentes sont presque toutes liées aux principales productions déjà mentionnées. Il s'agit de la vente sur pied, de l'association de production et du crédit fournisseur. L'offre de financement émane des commerçants collecteurs livreurs des fruits et légumes, des marâ̂chers sans terre et des fournisseurs

\footnotetext{
${ }^{1}$ DA : dinar algérien ; 100 dinars algériens = 1 euro.
}

d'intrants agricoles. Parmi les arboriculteurs enquêtés à Blida, 56 \% assurent le financement des charges d'acquisition des intrants (engrais et produits phytosanitaires) grâce aux avances des commerçants collecteurs et 37,9\% des maraîchers font appel à l'association de production. Pour Constantine, la céréaliculture, qui occupe $98 \%$ de la SAU de la wilaya, est la spéculation structurante de l'économie agricole de la région. L'élevage qui lui est associé, assez dynamique, joue le rôle d'activité secondaire. Classée parmi les régions céréalières potentielles, Constantine a été ciblée par le programme d'intensification des céréales dont les subventions constituaient, au moment de l'enquête, une source de financement importante pour les exploitations céréalières. Par ailleurs, les fournisseurs de services agricoles contribuent significativement au financement de la céréaliculture dans la wilaya, à travers les crédits de paiement des services agricoles (location de matériel). En effet, $72 \%$ des céréaliculteurs sans matériel de labour et qui recourent à la location, financent ce genre de services grâce au crédit de paiement. Ils sont près de $91 \%$ de céréaliculteurs à s'orienter vers ce mode de paiement pour la location de moissonneuses batteuses. De par l'importance du marché de la location du matériel, certains agriculteurs se sont spécialisés dans ce type de services et sont devenus de véritables entrepreneurs agricoles.

L'économie agricole à Djelfa est largement dominée par l'élevage pastoral dont l'importance $-3,4$ millions de têtes d'ovins - a favorisé le développement des marchés d'aliment du bétail et des services et produits vétérinaires. Poussés par la concurrence, les commerçants d'aliments et les vétérinaires jouent un grand rôle dans le financement del'activité d'élevage. Les crédits de paiement qu'ils offrent aux éleveurs en mal de liquidité, font partie intégrante des ressources de trésorerie de ces derniers. En effet, $44 \%$ des agriculteurs enquêtés dans la wilaya bénéficient de crédits de paiement de la part de leurs fournisseurs d'aliment du bétail et de services et produits vétérinaires. Une enquête récente (2010) auprès d'un fournisseur de services et intrants vétérinaires exerçant dans l'une des communes de la wilaya de Djelfa a confirmé l'importance de cette pratique. En effet, sur un chiffre d'affaire annuel de près de 4 millions de dinars, le vétérinaire enquêté déclare accorder à ses clients des crédits de paiement totalisant 730000 DA par an.
Dans les zones de montagne de la wilaya de Tlemcen, les potentialités agricoles sont limitées et aucune filière ne structure de manière significative l'économie agricole. Les exploitations sont plus petites et généralement très diversifiées, ne favorisant pas l'émergence d'importants marchés à l'amont ou à l'aval, dont les acteurs seraient les offreurs de financement informel. L'autofinancement est largement dominant et la contribution des acteurs de l'environnement économique et institutionnel est moins massive et systématique que dans les autres régions.

Ces différences dans les pratiques de financement informel dominantes s'expliquent par l'influence des données structurelles de l'économie agricole de chacune des régions. Une production agricole structurante, insérée dans une filière dynamique, façonne les opportunités de financement offertes aux agriculteurs. L'analyse des données de l'enquête montre les liens entre les caractères structurels de l'environnement des exploitations agricoles et les pratiques de financement informel des agriculteurs (figure 1).

La demande de financement de l'exploitation agricole est essentiellement déterminée par les caractéristiques du système de production. L'offre de financement informel peut provenir de trois types d'acteurs : les agriculteurs excédentaires en ressources productives, les partenaires économiques des agriculteurs, notamment les commerçants dans notre cas, et les membres des réseaux sociaux de l'agriculteur. Dans les régions à fort potentiel agricole, se développe autour de la spéculation agricole structurante, une économie de services qui, en fonction de la taille du marché et de sa structure, peut jouer un rôle plus au moins important dans le financement des exploitations agricoles. Les cas du commerce des fruits et légumes à Blida, des activités de prestations de services agricoles à Constantine ou celui du marché des services et produits vétérinaires à Djelfa, illustrent bien cette relation.

Par ailleurs, certaines catégories d'agriculteurs peuvent offrir des financements à d'autres. À Constantine, l'association de production est souvent initiée par des agriculteurs bien dotés en matériel agricole mais limités en foncier et qui cherchent à étendre leurs activités productives. Cette catégorie d'agriculteurs excédentaires en certaines ressources productives est plus importante dans les régions agricoles riches. 


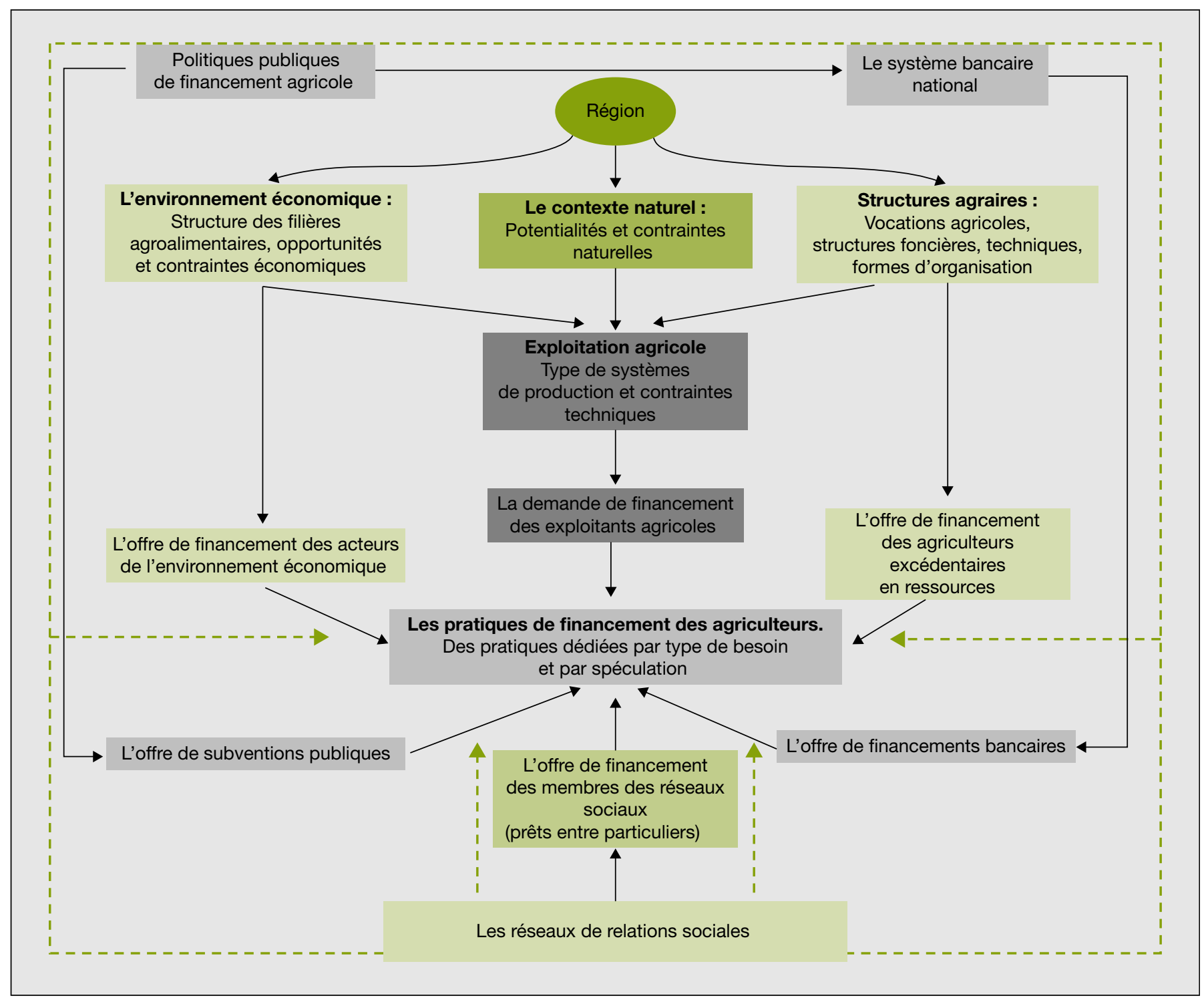

Figure 1. Représentation schématique des liens entre les facteurs contextuels et les pratiques de financement des agriculteurs.

Figure 1. Schematic representation of the links between contextual factors and farmers' funding practices.

Enfin, toutes les pratiques de financement informel sont enchâssées dans le tissu des rapports sociaux. Les agriculteurs peuvent faire partie de plusieurs réseaux, au sein desquels la mobilisation du capital social facilite l'accès à l'information et la construction de confiance.

\section{Conclusion}

Les résultats partiels exposés dans cet article démontrent l'existence et la relative importance du financement informel dans le fonctionnement de l'agriculture algérienne. Ils mettent en évidence les liens entre les caractéristiques des pratiques et les données de l'environnement naturel et socio-économique des exploitations agricoles. Cependant, la recherche dans laquelle s'insère cette étude a permis d'identifier les principales limites de ces pratiques (Daoudi et Bédrani, 2008). D'abord, la relative faiblesse des volumes de financement offerts : quelle que soit la pratique, l'offre de financement informel reste très en deçà des besoins des exploitations agricoles. Ensuite, l'offre de financement informel est limitée à des catégories de spéculations et à des besoins spécifiques, ne couvrant pas la diversité des besoins des agriculteurs. Enfin, cette offre est majoritairement inscrite dans le court terme et sa capacité à prendre en charge le financement des investissements productifs est limitée. De par ces limites, le financement informel ne peut donc pas contribuer de manière significative au développement et à la modernisation dont a besoin l'agriculture algérienne.

L'intérêt majeur de l'étude du financement informel réside dans le fait que son existence démontre que les risques de défaillance dans les transactions de financement agricole sont surmontables. En effet, l'existence même de ces pratiques laisse supposer que les agriculteurs et leurs partenaires sont parvenus à mettre 
en œuvre des mécanismes de gestion des risques de défaillance, efficaces et adaptés à leurs situations. L'inscription de la plupart des transactions de financement informel dans le cadre de relations économiques plus englobantes et généralement récurrentes peut être l'un des fondements des mécanismes de gestion des risques de défaillance dans ces transactions. La récurrence des transactions inscrit les rapports entre partenaires dans le long terme et réduit considérablement les comportements opportunistes

Les résultats de l'analyse de ces mécanismes pourraient alimenter la réflexion sur la réforme des systèmes du crédit agricole en Algérie et éventuellement dans les autres pays du Maghreb, par des idées qui les rapprocheraient davantage des spécificités de l'agriculture maghrébine et des logiques des agriculteurs. Car le défi de la modernisation de l'agriculture au Maghreb passe inévitablement par le développement de marchés financiers ruraux efficaces.

\section{Références}

Adams D, Fitchett D. Finance informelle dans les pays en développement. Lyon : Presses Universitaires de Lyon, 1994.
Akesbi N, Benatya D, El Aoufi N. L'agriculture marocaine à l'épreuve de la libéralisation. Rabat: Economie Critique, 2008.

Andersson C, Holmgren E, MacGregor, Stage J. Giving credit to the microlenders. Formal microlending, credit constraints and adverse selection: a case study of shrimp farmers in Bangladesh. Discussion Paper 08-02. Londres: International Institute for Environment and Development, 2008.

Auditnet Tunisie. Financement des projets agricoles. Tunis: Audinet Tunisie, 2007. www. investir-en-tinisie.net/news/article.php?id=1268

Banque mondiale. La finance pour tous? Politiques et écueils du développement. Washington: Banque mondiale, 2007. www.banquemondiale. org/pulications/pub-landing.htm\#finance-afrique

Ben El Ahmar M. Les contraintes de financement de I'agriculture au Maroc. Revue H.T.E 2003; (162) : 65-6.

Conseil national économique et social (CNES). Stratégie de développement de l'agriculture. Alger: CNES, 2003. www.cnes.dz/cnesdoc/plein $\% 2018 /$ SGDA31.htm

Daoudi A, Bédrani S. Le financement informel des exploitations agricoles en Algérie : un essai de caractérisation des principales pratiques. Cahiers du CREAD 2008 ; (85-86) : 109-34.

Daoudi A, Bedrani S. Le financement non institutionnel dans l'agriculture: quelques résultats d'une enquête rapide. Revue Recherche Agronomique/INRAA 2002 ; (1) : 77-89.

Henni A. Le cheikh et le patron. Usage de la modernité dans la reproduction de la tradition. Alger: OPU, 1993.
Krugman P. The new economic geography where are we?. Communication présentée au symposium international sur "Globalization and Regional Integration - from the viewpoint of Spatial Economics" organisé par IDE-JETRO, 2004. www.ide.go.jp/English/Events/Sympo/pdf/ krug_summary.pdf

Mesli ML. L'agronome et la terre. Alger : éditions Alpha, 2007.

Mesli ML. Le financement de I'agriculture : aspects économiques et contraintes sociales. In: Benachenhou A. Du budget au marché. Alger: éditions Alpha, 2004.

Maksimovic V, Demirguc-Kunt A, Ayyagari M. Formal versus informal finance: evidence from China. Policy Research Working Peppers Series. Washington (DC) : Banque mondiale, 2008.

Morvant-Roux S. Quelle microfinance pour l'agriculture des pays en développement? Synthèse $d u$ colloque organisé par FARM, 2008. www.fondation-farm.org/IMG/pdf/Farm_synthese_microfinance_.pdf

Recensement général de l'agriculture (RGA). Rapport général des résultats définitifs. Alger: ministère de I'Agriculture et du Développement rural, 2003.

Ruan J, Zhang X. Finance and Cluster-Based Industrial Development in China. Discussion Paper de I'IFPRI. Washington (DC) : IFPRI, 2008.

Servet JM. Épargne et liens sociaux. Étude comparée d'informalités financières. Paris : Association d'économie financière, 1995.

Tsai Kellee S. Imperfect Substitutes: The local political economy of informal finance and microfinance in rural China and India. World Development 2004: 1487-507.

Venet $\mathrm{B}$. Libéralisation financière et développement économique : une revue critique de la littérature. $R E F 1994 ; 29: 87-111$. 